EPJ manuscript No.

(will be inserted by the editor)

\title{
Low temperature thermodynamics of inverse square spin models in one dimension
}

\author{
W. Hofstetter ${ }^{1}$ and W. Zwerger ${ }^{2}$ \\ 1 Theoretische Physik III, Universität Augsburg, 86135 Augsburg, Germany \\ ' 2 Sektion Physik, Ludwig-Maximilians-Universität München, Theresienstr. 37, 80333 München, Germany
}

April 1998

\begin{abstract}
We present a field-theoretic renormalization group calculation in two loop order for classical $O(N)$-models with an inverse square interaction in the vicinity of their lower critical dimensionality one. The magnetic susceptibility at low temperatures is shown to diverge like $T^{-a} \exp (b / T)$ with $a=(N-2) /(N-1)$ and $b=2 \pi^{2} /(N-1)$. From a comparison with the exactly solvable Haldane-Shastry model we find that the same temperature dependence applies also to ferromagnetic quantum spin chains.
\end{abstract}

PACS. $75.10 . \mathrm{Hk}$

\section{Introduction}

The investigation of long range forces in statistical mechanics has a considerable history, including studies on the basic requirements for a proper thermodynamic limit 1] or on the existence of phase transitions even in onedimensional systems. For classical spins, it has been known for some time [2] that ferromagnetic models with a pairinteraction decaying like $n^{-(1+\sigma)}(\sigma>0$ is necessary for a proper thermodynamic limit) exhibit no transition if $\sigma>1$. Choosing $\sigma<1$ however, it turns out there is indeed a phase transition even in one dimension both for Ising models [3 as well as for systems with a continuous symmetry 沟. The borderline case $\sigma=1$ of an inverse square interaction is thus of particular interest. For Ising spins an early conjecture by Thouless [5] that this model exhibits a peculiar continuous transition with a finite jump of the order parameter was finally proven rigorously by Fröhlich and Spencer [6]. In the case of a continuous symmetry like the $X Y$ - or Heisenberg-models with inverse ' square interaction, Simon has shown [7] that no symmetry breaking appears here, suggesting that the borderline interaction exhibiting a Kosterlitz-Thouless type transition is $(\ln n) / n^{2}$. An alternative proof that the inverse square $X Y$-model in one dimension exhibits no long range order was later given by Simanek [8], using the classical version of the Mermin-Wagner theorem.

A quantitative calculation of the low temperature susceptibility of long range ferromagnetic spin models was given by Kosterlitz [9] within a momentum shell renormalization group (RNG) up to one loop order. In particular the susceptibility was found to diverge exponentially like $\exp \left(2 \pi^{2} /(N-1) T\right)$ in the case $\sigma=1$ of an inverse square interaction for models with a continuous symmetry, where only the trivial fixed point at $T=0$ describing a fully ordered ground state exists. Independently, in a brief note, Brézin, Zinn-Justin and Le Guillou [10] presented the results of a two loop field-theoretic RNG of the $O(N)$ model with interaction proportional to $n^{-(1+\sigma)}$. They determined the critical exponents up to order $(d-\sigma)^{2}$ in the vicinity of the lower critical dimension $d_{c}=\sigma$. Here we present a detailed version of the field-theoretic two loop calculation which essentially follows their ideas. However, it uses a somewhat different method to determine the renormalization factors and, moreover, concentrates on the behaviour in the marginal case $d=\sigma=1$.

The study of one-dimensional classical spin models with long range interactions is interesting not only from a pure statistical mechanics point of view, but is also relevant for actual physical problems. In fact the inverse square Ising-model is closely related to the well-known Kondo-problem of a magnetic impurity locally coupled to the conduction electron spin density [11]. A more recent application of the same model is the two state system with ohmic dissipation, arising e.g. in the problem of quantum coherence between macroscopically different states [12,13]. For the inverse square $X Y$-case it turns out that the problem of strong tunneling in a metallic single-electron-box can be treated by calculating the free energy of the classical spin model in the presence of twisted boundary conditions [14]. It was the latter problem which motivated our present work, whose intention is in fact twofold: First of all, we give a detailed account of the two-loop RNG of the $O(N)$-model with inverse square interaction which has obviously not been done in the literature so far. In addition to that we discuss the corresponding $S=1 / 2$ quantum spin chain. In particular, it is found that the low temperature behaviour of the susceptibility is essentially identical with that for classical spins. 


\section{RNG in two-loop order}

\section{a) Model and Definitions}

We start with a model of classical unit spins $\mathbf{S}(n)$ with $N$ components which are placed on a ring with $L$ sites, thus enforcing periodic boundary conditions. The interaction energy in an external magnetic field $H$ along the $N$-direction is given by

$$
H_{c l}=-\sum_{n \neq n^{\prime}}\left[d\left(n-n^{\prime}\right)\right]^{-2} \mathbf{S}(n) \mathbf{S}\left(n^{\prime}\right)-H \sum_{n} S^{N}(n)
$$

with

$$
d(n)=\frac{L}{\pi} \sin \frac{\pi|n|}{L}
$$

the chord distance between spins which are $n$ sites apart. The associated partition function can then be written as

$$
\mathcal{Z}=\int \prod_{n} d \mathbf{S}(n) \delta\left(\mathbf{S}^{2}(n)-1\right) \exp \{-S(T, H, \mathbf{S}(n))\} .
$$

with an action

$$
\mathcal{S}=\frac{1}{2 T} \sum_{n \neq n^{\prime}} \frac{\left(\mathbf{S}(n)-\mathbf{S}\left(n^{\prime}\right)\right)^{2}}{\left(d\left(n-n^{\prime}\right)\right)^{2}}-\frac{H}{T} \sum_{n} S^{N}(n) .
$$

Here $\int d \mathbf{S}(n) \delta\left(\mathbf{S}^{2}(n)-1\right)$ denotes an integration over all orientations of a classical $N$-component unit vector $\mathbf{S}(n)$. As is known from the work cited above, this model exhibits a continuous phase transition to an ordered phase below a critical temperature $T_{c} \sim \epsilon /(N-1)$ for noninteger dimensionality $d=1+\epsilon(\epsilon>0)$. In order to study the behavior near the lower critical dimensionality one, we can therefore parametrize the spin $\mathbf{S}$ according to a low temperature expansion

$$
\mathbf{S}(x)=\left(\boldsymbol{\Pi}(x), \sqrt{1-\Pi^{2}(x)}\right) .
$$

The $\Pi_{i}(i=1, \ldots, N-1)$ are Goldstone modes corresponding to the fluctuations around the ordered state with $S_{N}=1$ where all spins point in the $N$-direction. The generating functional is now given by

$$
\mathcal{Z}=\int \prod_{n} \frac{d \boldsymbol{\Pi}(n)}{\sqrt{1-\Pi^{2}(n)}} \exp \{-S(T, H, \Pi(n))\} .
$$

The term in the integration measure

$$
\prod_{n} \frac{1}{\sqrt{1-\boldsymbol{\Pi}^{2}(n)}}=\exp \left\{-\frac{1}{2} \sum_{n} \ln \left(1-\boldsymbol{\Pi}^{2}(n)\right)\right\}
$$

is due to the $\delta$-function constraint and ensures the $O(N)$ symmetry of the model, which appears to be broken by the above parametrization. In a continuum notation $\sum_{n} \rightarrow$ $a^{-d} \int d^{d} x$ with $a$ the lattice constant and

$$
\prod_{n} \frac{1}{\sqrt{1-\boldsymbol{\Pi}^{2}(n)}}=\exp \left\{-\frac{1}{2} a^{-d} \int d^{d} x \ln \left(1-\boldsymbol{\Pi}^{2}(x)\right)\right\}
$$

In the following, we will work in the dimensional regularization scheme, where

$$
a^{-d}=\int^{\Lambda} \frac{d^{d} q}{(2 \pi)^{d}}=0 .
$$

We can therefore neglect the measure term (see 15]).

In order to generate correlation functions of the $\Pi$ fields, we introduce additional source fields according to

$$
\mathcal{S} \mapsto \mathcal{S}-\int d x \mathbf{J}(x) \mathbf{\Pi}(x)
$$

The quantities which are most conveniently calculated diagrammatically are the irreducible vertex functions $\Gamma^{(n)}$. Their generating functional $\Gamma[\overline{\mathbf{\Pi}}, H]$ is formally obtained from $\ln \mathcal{Z}[\mathbf{J}, H]$ by a Legendre transformation with respect to $\mathbf{J}$ where $\overline{\boldsymbol{\Pi}}$ is the corresponding classical field. In our calculation, we will use the two point function

$$
\Gamma^{(2)}(x, y)=\frac{\delta^{2} \Gamma}{\delta \bar{\Pi}(x) \delta \bar{\Pi}(y)} .
$$

As in the case of the non-linear $\sigma$-model with nextneighbour coupling [15,16] it can be shown that the model is renormalizable by introducing two renormalization factors $Z_{t}$ and $Z_{\pi}$ rescaling the temperature and the field $\Pi$. These factors must be adjusted in such a way that the renormalized $N$-point functions

$$
\Gamma_{R}^{(N)}=Z_{\pi}^{\frac{N}{2}} \Gamma^{(N)}
$$

remain finite in terms of the renormalized parameters

$$
\begin{gathered}
t=\kappa^{\epsilon} Z_{t}^{-1} T \\
h=Z_{t}^{-1} Z_{\pi}^{\frac{1}{2}} H .
\end{gathered}
$$

Below we will see that the choice $Z_{t}=Z_{\pi} \equiv Z$ with a single independent renormalization constant $Z$ is possible.

\section{b) Details of the two-loop-calculation}

In order to calculate the $Z$-factors introduced above to the order $t^{2}$, we first rewrite the action in momentum representation:

$$
\begin{aligned}
\mathcal{S}= & \frac{1}{T} \int \frac{d^{d} q}{(2 \pi)^{d}}(|q|+H)\left[\frac{\boldsymbol{\Pi}(q) \boldsymbol{\Pi}(-q)}{2}+\frac{\boldsymbol{\Pi}^{2}(q) \boldsymbol{\Pi}^{2}(-q)}{8}\right. \\
& \left.+\frac{\boldsymbol{\Pi}^{2}(q)\left(\boldsymbol{\Pi}^{2}\right)^{2}(-q)}{16}\right]+O\left(\left(\boldsymbol{\Pi}^{2}\right)^{4}\right),
\end{aligned}
$$

where we have rescaled $H \mapsto 2 \pi H$ and $T \mapsto(2 \pi)^{d} T$. The free propagator corresponding to the quadratic term has the form

$$
\begin{aligned}
\left\langle\Pi^{\alpha}(p) \Pi^{\beta}(q)\right\rangle & =\delta_{\alpha \beta} \delta(p+q) G_{0}^{\alpha}(p) \\
& =(2 \pi)^{d} \delta_{\alpha \beta} \delta(p+q) \frac{T}{|p|+H} .
\end{aligned}
$$



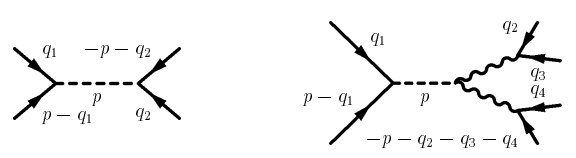

Fig. 1. Diagrammatic representation of the four- and six-point interaction terms.

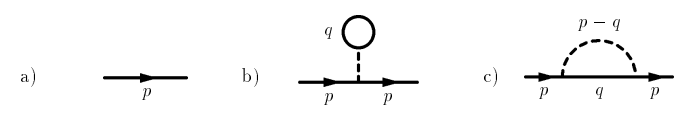

Fig. 2. Free propagator (a)) and one-loop contributions to $\Gamma^{(2)}(\mathrm{b})$ and c)).

with $\alpha, \beta=1, \ldots, N-1$. In addition, $\mathcal{S}$ contains two interaction terms which contribute up to two-loop order. These are shown in fig. (11) and correspond to a four- and a six-point-interaction. We will renormalize the model using the two-point function $\Gamma^{(2)}$. The relevant diagrams up to two-loop order are shown in fig. (2) and (3). In a short hand notation, the two point function in terms of these diagrams is given by

$$
\begin{aligned}
\Gamma^{(2)}(k)= & a)+b)+c)-d)-e)-f(-g)-h)-i) \\
& +j)+k)+l)+m)+n)+o(+p)+q) .(17)
\end{aligned}
$$

Each one of these diagrams is proportional to $T^{L-1}$ where $L$ is the number of loops. Several of them contain divergences for $\epsilon \rightarrow 0$. In the minimal subtraction scheme, we want to absorb these divergences in the two renormalization constants $Z_{t}$ and $Z_{\pi}$. After renormalization, $\Gamma_{R}^{(2)}$ should be an analytic function of the variables $h, t$ and $p$.

For the action (15) we note that the $p$-dependent parts of the diagrams are finite. To one-loop order, for instance, only the second diagram (labeled by c)) depends on the external momentum

$$
\int \frac{d^{d} q}{(2 \pi)^{d}} \frac{1+|p-q|}{1+|q|}=\int \frac{d^{d} q}{(2 \pi)^{d}} \frac{|p-q|-|q|}{1+|q|} .
$$

Evidently this integral is finite for $d<2$. Because of

$$
\Gamma_{R}^{(2)}(p)=\frac{Z_{\pi}}{Z_{t}} \kappa^{\epsilon} \frac{|p|+Z_{t} Z_{\pi}^{-\frac{1}{2}} h}{t}+\text { higher order terms }
$$

the coefficient of $|p|$ in the inverse propagator is renormalized by $Z_{\pi} / Z_{t}$. As is evident from the one-loop term (18), this prefactor is already finite. Therefore the choice

$$
Z_{\pi}=Z_{t} \equiv Z
$$

is possible, leaving only one independent renormalization constant. In fact this relation is valid to arbitrary loop order, as was shown by Brézin, Zinn-Justin and Le Guillou [10]. As a consequence, we can take the external momentum $p$ to be zero in the following. One further simplification is due to the fact that in dimensional regularization

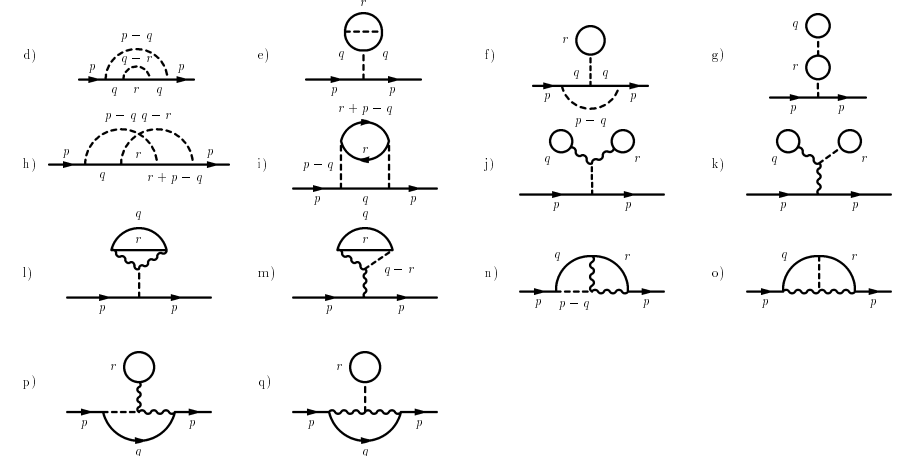

Fig. 3. Two-loop contributions to $\Gamma^{(2)}$.

"empty" integrals of the type $\int d^{d} q$ are equal to zero. This implies that the contributions of the diagrams in c), h), n) and $\mathrm{p}$ ) vanish. Moreover, several contributions turn out to be identical and therefore cancel: this applies to the diagrams d) and o) which are given by

$$
T H^{2 d-1} \iint \frac{d^{d} q d^{d} r}{(2 \pi)^{2 d}} \frac{1+|q-r|}{(1+|q|)(1+|r|)} .
$$

Similarly the diagrams i) and $\mathrm{m}$ ) which give a contribution

$$
\frac{N-1}{2} T H^{2 d-1} \iint \frac{d^{d} q d^{d} r}{(2 \pi)^{2 d}} \frac{1+|q-r|}{(1+|q|)(1+|r|)}
$$

are equal and therefore cancel in $\Gamma^{(2)}$. The remaining expression to be evaluated is

$$
\left.\left.\left.\left.\left.\left.\left.\left.\left.\Gamma^{(2)}(0, H)=a\right)+b\right)-e\right)-f\right)-g\right)+k\right)+j\right)+l\right)+q\right) .
$$

In order to extract the singular parts of the integrals, we use the asymptotic expansions

$$
\int \frac{d^{d} q}{(2 \pi)^{d}} \frac{1}{1+|q|}=K_{d}\left(-\frac{1}{\epsilon}+o(\epsilon)\right)
$$

and

$$
\int \frac{d^{d} q}{(2 \pi)^{d}} \frac{1}{(1+|q|)^{2}}=K_{d}\left(1+o\left(\epsilon^{2}\right)\right)
$$

where the constant

$$
K_{d}=\frac{2}{(4 \pi)^{d / 2} \Gamma(d / 2)}
$$

results from the $d$-dimensional angular integration. The identities (24) and (25) allow to extract the leading singularities in $\epsilon$ for all contributions to (23) except e). By a detailed asymptotic analysis of the corresponding integral, the following singular contributions of this diagram can be obtained:

$$
\begin{aligned}
e) & =\iint \frac{d^{d} q d^{d} r}{(2 \pi)^{2 d}} \frac{1+|r-q|}{(1+|q|)^{2}(1+|r|)} \\
& =\frac{N-1}{2} T H^{1+2 \epsilon} K_{d}^{2}\left(\frac{1}{2 \epsilon^{2}}+\frac{1}{2 \epsilon}+o(1)\right) .
\end{aligned}
$$


Neglecting nonsingular parts, the resulting two-point function is therefore finally given by

$$
\begin{aligned}
\Gamma^{(2)}(0, H)= & \frac{H}{T}-H^{1+\epsilon} \frac{N-1}{2 \epsilon}+T H^{1+2 \epsilon} \frac{(N-1)(N-2)}{4 \epsilon} \\
& +T H^{1+2 \epsilon} \frac{3(N-1)^{2}}{8 \epsilon^{2}}
\end{aligned}
$$

where we have absorbed the angular integration term $K_{d}$ in the temperature according to

$$
T \mapsto T K_{d}^{-1} \quad, \quad \Gamma^{(2)} \mapsto \Gamma^{(2)} K_{d}
$$

At this stage we can now introduce renormalized parameters $h$ and $t$ as defined in (13) and (14). Taking $\kappa=1$ for convenience, we obtain

$$
\begin{aligned}
\Gamma_{R}^{(2)}(0, h)= & \frac{Z^{\frac{1}{2}} h}{t}-\frac{N-1}{2 \epsilon} Z^{1+\frac{1+\epsilon}{2}} h^{1+\epsilon} \\
& +t h^{1+2 \epsilon} \frac{(N-1)(N-2)}{4 \epsilon}+t h^{1+2 \epsilon} \frac{3(N-1)^{2}}{8 \epsilon^{2}}
\end{aligned}
$$

where terms of order $t^{2}$ have been neglected. Using the ansatz

$$
Z=1+a_{1}(\epsilon) t+a_{2}(\epsilon) t^{2}+o\left(t^{3}\right)
$$

this can be written as

$$
\begin{aligned}
& \Gamma_{R}^{(2)}(0, h)=\frac{h}{t}+h\left(\frac{1}{2} a_{1}-\frac{N-1}{2 \epsilon}\right)+t h\left(\frac{4 a_{2}-a_{1}^{2}}{8}\right. \\
& \left.-\frac{(N-1)\left((3+\epsilon) a_{1}-(N-2)\right)}{4 \epsilon}+\frac{3(N-1)^{2}}{8 \epsilon^{2}}\right) \\
& +t \ln h\left(\frac{3(N-1)^{2}}{4 \epsilon}-\frac{(N-1)}{2} \frac{(3+\epsilon)}{2} a_{1}\right)
\end{aligned}
$$

where we have neglected terms which are not singular in $\epsilon$. The coefficients $a_{1}$ and $a_{2}$ are now chosen such as to eliminate the poles of order $\epsilon^{-1}$ and $\epsilon^{-2}$. This is achieved by

$$
a_{1}=\frac{N-1}{\epsilon}
$$

and

$$
a_{2}=\frac{(N-1)^{2}}{\epsilon^{2}}+\frac{(N-1)}{2 \epsilon},
$$

thus rendering the renormalized two point function finite. The renormalization constant $Z$ is therefore given by

$$
Z=1+\frac{N-1}{\epsilon} t+\left(\frac{(N-1)^{2}}{\epsilon^{2}}+\frac{N-1}{2 \epsilon}\right) t^{2}+O\left(t^{3}\right)
$$

From this result we can now determine the beta function

$$
\beta(t) \equiv-\kappa \frac{\partial t}{\partial \kappa}
$$

measuring the variation of the renormalized coupling under variation of the momentum scale, keeping the bare parameters $T$ and $H$ fixed. According to (13), we can write

$$
\beta(t)=-\epsilon t\left(1+t \frac{d \ln Z}{d t}\right)^{-1} .
$$

Using the result (35) this immediately leads to

$$
\beta(t)=-\epsilon t+(N-1) t^{2}+(N-1) t^{3}+O\left(t^{4}\right) .
$$

For $\epsilon>0$, the renormalization flow has a non trivial fixed point corresponding to a phase transition at finite temperature. The associated critical behaviour has been discussed by Brézin et al. [10]. Here we are interested in the one-dimensional case, where only the trivial fixed point at $t=0$ describing the fully ordered ground state exists. During the calculation we have rescaled the coupling $T$ twice. Inserting the original temperature according to $T \rightarrow T / 2 \pi^{2}$ we get the following equation for the flow of $T$ under the reduction of the cutoff $\Lambda \rightarrow \Lambda e^{-l}$ :

$$
\frac{d T}{d l}=(N-1) \frac{T^{2}}{2 \pi^{2}}+(N-1) \frac{T^{3}}{\left(2 \pi^{2}\right)^{2}}+O\left(T^{4}\right) .
$$

Integrating this equation between an initial temperature $T$ very close to zero and a final value $T=O(1)$, the corresponding length rescaling factor behaves like

$$
\xi(T) \sim T^{\frac{1}{N-1}} \exp \left(\frac{2 \pi^{2}}{(N-1) T}\right) .
$$

Obviously $\xi(T)$ plays the role of a "correlation length", however it is important to point out that there can be no exponential decay in models with long range interactions. In fact, using Lieb-Simon-type inequalities, it can be shown that the spin-spin correlation function

$$
g(n)=\langle\mathbf{S}(n) \mathbf{S}(0)\rangle
$$

asymptotically decays like $n^{-2}$ for arbitrary temperatures [17, 18. In spite of the absence of an exponential decay, within a standard scaling hypothesis the behavior of $g(n)$ near the critical point at $T=0$ is described by a universal function $f(n)$ with $f(0)=1$ and $f(|n| \rightarrow \infty)=f_{\infty} / n^{2}$, $f_{\infty}=O(1)$, such that

$$
g(n, T \rightarrow 0)=f\left(\frac{n}{\xi(T)}\right)
$$

with $\xi(T)$ given by $(40)$. As a result the magnetic susceptibility

$$
\chi=\beta\left(1+2 \sum_{n=1}^{\infty} g(n)\right)
$$

is finite for any $T$ and scales like

$$
\chi(T) \sim T^{-\frac{N-2}{N-1}} \exp \left(\frac{2 \pi^{2}}{(N-1) T}\right)
$$

at low temperatures. The two loop calculation thus uniquely determines both the exponent and the $T$ dependence of the prefactor of the magnetic susceptibility as $T \rightarrow 0$. 


\section{Discussion}

In this work we have calculated the low temperature susceptibility of classical $O(N)$ spin models with an inverse square interaction. Now it is interesting to compare our results with the corresponding ferromagnetic quantum spin models. In the case of a Heisenberg model

$$
\hat{H}=-\sum_{n \neq n^{\prime}} J\left(n-n^{\prime}\right) \hat{\mathbf{S}}_{n} \hat{\mathbf{S}}_{n^{\prime}}-H \sum_{n} \hat{S}_{n}^{z}
$$

with interaction $J(n)=d^{-2}(n)$, the exact low temperature susceptibility in the case $S=1 / 2$ was calculated by Haldane 19. It is given by

$$
\chi(T)=\frac{1}{4 \sqrt{\pi}} T^{-\frac{1}{2}} \exp \left(\frac{\pi^{2}}{4 T}\right) .
$$

and thus exhibits precisely the same temperature dependence as our result (44) for the classical case with $N=3$ (note that we have chosen units in which $J=1$ and our temperature differs from the one in ref. [19 by a trivial factor two arising from $\left.\sum_{n \neq n^{\prime}}=2 \sum_{n<n^{\prime}}\right)$. In fact the only difference is in the numerical factor which appears in the exponent. On a qualitative level the exponential divergence of $\chi(T)$ in the case of long range interactions can be understood from a simple spin wave calculation around the perfectly ordered ferromagnetic ground state. For a general spin $S$, the Hamiltonian (45) leads to a dispersion relation of magnons which has the form

$$
\epsilon(k)=4 S \sum_{n>0} J(n)(1-\cos (k n))+H .
$$

An equilibrium distribution of noninteracting magnons leads to the standard reduction in the dimensionless magnetization

$$
m(T, h)=1-\frac{1}{S} \int \frac{d k}{2 \pi} \frac{1}{e^{\beta \epsilon(k)}-1} .
$$

For the Haldane-Shastry model with $J(n)=d^{-2}(n)$, the magnon dispersion (47) is given by

$$
\epsilon(k)=S\left(2 \pi|k|-k^{2}\right)+H
$$

(see also 19]). The associated reduction in magnetization in the limit $H, T \rightarrow 0$ then behaves like

$$
\delta m(T, H)=\frac{T}{2(\pi S)^{2}} \ln \frac{2 \pi S}{H}
$$

The condition $\delta m=b$ with a constant $b$ of order one then defines a field $H(T)$ which qualitatively determines the zero field susceptibility via

$$
\chi(T) \approx \frac{1}{H(T)} \sim \exp \frac{2 b(\pi S)^{2}}{T} .
$$

Choosing $b=1 / 2$, this result agrees precisely with the exact solution (46) for $S=1 / 2$, while the exponent in the susceptibility of the classical spin model with unit spin is obtained by formally setting $S=1$. A modified spin wave theory and Schwinger boson techniques have been applied to the Haldane-Shastry model by Nakano and Takahashi 2021 .

For short range interactions where $J(n)$ decays faster than $n^{-3}$, the magnon dispersion has the usual form

$$
\epsilon(k \rightarrow 0)=D k^{2}+H .
$$

It is then straightforward to see that the above spin wave calculation leads to a susceptibility diverging like $\chi(T) \sim S^{2} / T^{2}$. This behavior is indeed found from the exact solution of both the nearest neighbor $S=1 / 2$ case [22] as well as for the corresponding classical Heisenberg model [23]. We thus expect that for any ferromagnetic spin model with a fully ordered ground state the temperature dependence of the susceptibility at low $T$ is independent of whether the spins are classical or quantum mechanical.

In the case of an $X Y$-model $(N=2)$, the ground state for the $S=1 / 2$ chain with inverse square interaction has again a very simple structure, as shown by Haldane 24. It would thus be interesting to check whether in this case the low temperature susceptibility again diverges like that of the classical model with $N=2$, i.e. $\chi(T) \sim \exp \left(2 \pi^{2} / T\right)$.

\section{References}

1. See e.g. R.B. Griffiths in "Phase Transitions and Critical Phenomena", Vol. 1, Ed. C. Domb and M.S. Green, Academic Press, London 1971.

2. D. Ruelle, Comm. Math. Phys. 9, 267 (1968).

3. F.J. Dyson, Comm. Math. Phys. 12, 91 (1969).

4. J. Fröhlich, R. Israel, E. Lieb and B. Simon, Comm. Math. Phys. 62, 1 (1978).

5. D.J. Thouless, Phys. Rev. 187,732 (1969).

6. J. Fröhlich and T. Spencer, Comm. Math. Phys. 84, 87 (1982).

7. B. Simon, J. Stat. Phys. 26, 307 (1981).

8. E. Simanek, Phys. Lett. 119A, 477 (1987). Note, however, that the prediction in this paper of an infinite susceptibility at low temperatures is not correct as is shown here.

9. J. M. Kosterlitz, Phys. Rev. Lett. 37, 1577 (1976).

10. E. Brézin, J. Zinn-Justin und J. C. Le Guillou, J. Phys. A 9, L119 (1976).

11. P.W. Anderson, G. Yuval and D.R. Hamann, Phys. Rev. B1, 4464 (1970), P.W. Anderson and G. Yuval, J. Phys. C4, 607 (1971).

12. A.J. Leggett, S. Chakravarty, A.T. Dorsey, M.P.A. Fisher, A. Garg and W. Zwerger, Rev. Mod. Phys. 59, 1 (1987); ibid. 67, 725 (1995) (erratum).

13. For recent work see S. Chakravarty and J. Rudnick, Phys. Rev. Lett. 75, 501 (1995); F. Lesage and H. Saleur, condmat 9712019 .

14. W. Hofstetter and W. Zwerger, Phys. Rev. Lett. 78, 3737 (1997).

15. D. J. Amit, Field Theory, the Renormalization Group, and Critical Phenomena (World Scientific, Singapore 1984).

16. J. Zinn-Justin, Quantum Field Theory and Critical Phenomena (Oxford University Press, Oxford 1996).

17. W. Zwerger and M. Scharpf, Z. Phys. B85, 421 (1991). 
18. H. Spohn and W. Zwerger, unpublished. The proof uses Gaussian domination, which is known only for $N \leq 4$.

19. F.D.M. Haldane, Phys. Rev. Lett. 66, 1529 (1991).

20. H. Nakano and M. Takahashi, Phys. Rev. B50, 10331 (1994).

21. H. Nakano and M. Takahashi, J. Phys. Soc. Jpn. 63, 4256 (1994).

22. M. Takahashi, Phys. Rev. B40, 2494 (1989).

23. M. Fisher, Am. J. Phys. 32, 343 (1964).

24. F.D.M. Haldane, Phys. Rev. Lett. 60, 635 (1988). 